\title{
PFA: Program for the quantitative assessment of cell metabolism by spectral data analysis
}

\author{
Chris Orr $^{1,}{ }^{*}$, Stephen Scott ${ }^{1},{\text { Fouad } \text { Kandeel }^{1}}$ and Kevin Ferreri ${ }^{1}$ \\ ${ }^{1}$ Southern California Islet Cell Resources Center, Department of Diabetes, Endocrinology, and Metabolism, Beckman Research Institute of the \\ City of Hope, Duarte, CA; Chris Orr* - E mail: rahsaan26@hotmail.com; * Corresponding author \\ received September 02, 2008; accepted September 17, 2008; published October 18, 2008
}

\begin{abstract}
:
Assessment of mitochondrial oxidative metabolism has wide-ranging importance, from pharmacokinetic analysis to studies in cell viability and apoptosis. Here, we present the Perfusion File Analyzer (PFA) application for the real-time analysis of spectral data to measure cytochrome c reduction, cytochrome a3 reduction, and other parameters important to cellular metabolism, which are collected during tissue perfusion experiments. Our current efforts are focused on quantitating changes in mitochondrial function by normalizing baseline drift of spectral data while addressing two major challenges: (1) a lack of real-time feedback from the system when aiming is compromised, and (2) an inability to adjust calculated data in the event of spectral shift. PFA has been developed to address these issues, and is currently used for quality assessment of human islets prior to clinical transplantation.
\end{abstract}

Keywords: oxidative metabolism; cell; data analysis; pharmacokinetics analysis

\section{Background:}

As the primary mechanism of energy production, mitochondrial function is critical to normal cell function. We have been employing a previously developed (3) perfusion system to collect oxygen consumption, insulin secretion, and spectral data from human islets in response to glucose. Stimulation with glucose results in the reduction of cytochrome $\mathrm{c}$ and cytochrome $\mathrm{a}_{3}$, which absorb at 551 and $605 \mathrm{~nm}$ respectively, and is subsequently quantified by comparison with fully, oxidized and fully reduced states. Data for fully oxidized and reduced cytochrome is obtained using the electron transport chain inhibitors Antimycin A, which blocks the transport of electrons upstream of the cytochromes, and $\mathrm{KCN}$, which blocks electrons downstream of the cytochromes [1]. Data is then calculated as the percent reduced cytochrome relative to the fully reduced state. Previously, these calculations utilized macros in Excel software (Microsoft, Redmond, WA) resulting in a time intensive process for computing only 2 wavelengths per experiment, was prone to operator copy/paste and logistical errors, and took between 1-2 hours to complete. We have developed a program, perfusion file analyzer (PFA), to address these issues and provide greater flexibility in the analysis of the data (screenshot in Figure $1 \mathrm{a}$ and $\mathrm{b}$ ).

\section{Methodology: \\ Software input and output}

The PFA program is written in the JAVA programming language and utilizes the Apache POI API (http://poi.apache.org/). Raw data generated from the

ISSN 0973-2063 (online) 0973-2063 (print)

Bioinformation 3(2): 65-67 (2008) spectrometer software (OOIBase32 Spectrometer Operating Software, Ocean Optics, Dunedin, FL) are saved as SCOPE files which are read directly by PFA as the files are created. PFA calculates the second derivative of the absorbance data (Abs") with respect to wavelength $(v)$ as described in equation 1 (see supplementary material) as described elsewhere [2].

During the perfusion experiment, the program calculates the second derivative of the absorbance and plots it against wavelength in real-time. The unique profile of the cells in this graph provides immediate feed-back for the operator, especially when the spectral data is compromised from issues such as the introduction of bubbles in the system, or a shift in the location of the cell sample. The graph includes "aiming brackets" that assist in the initial adjustment of the spectrophotometer, and detects the spectral shift if the spectrometer is not properly aimed at the cell pellet, or if the pellet moves during the experiment.

At the conclusion of the run, second derivative post run data is also generated, comprised of a matrix of absorbance data spanning several hundred wavelengths plotted against time which the user can scroll through manually. The ability of PFA to present the data as a list of Abs" arrays helps the operator to visually detect and resolve data compromised by poor aiming or disruption of the pellet. The program offers roll over data for each data point on the graph, which can then be selected by the user to define the range of fully oxidized and reduced values for normalization of data. Based on these user selected values, the program recalculates and plots the data as percent reduction of 65 


\section{www.bioinformation.net}

cytochrome over time using the equation 2 (see supplementary material) as described elsewhere [3].

Additionally, in the post-run analysis the program will import oxygen consumption data from the lifetime spectrofluorometer files (Oxygen Enterprises, Philadelphia, PA) and also any Microsoft Word document (Microsoft Office, Microsoft, Redmond, WA) with notes associated with the experiment and display them both within the application window.

In time tests, PFA analyzed over 400 individual spectrophotometer files per experiment in an average of 5 seconds (Pentium 4, $2.8 \mathrm{GHz}$; Intel, Santa Clara, CA) for post run analysis of data for each replicate, which previously took between 1-2 hours to complete using Excel macros. By automating the process of data calculation and simplifying the data acquisition process by providing immediate user feedback, PFA not only provides a more comprehensive tool for spectral data analysis, but can help the operator to avoid failed experiments by displaying aiming status throughout the course of the experiment. The result is a more robust, expansive, and accurate environment with which to analyze spectral data for cell metabolism.

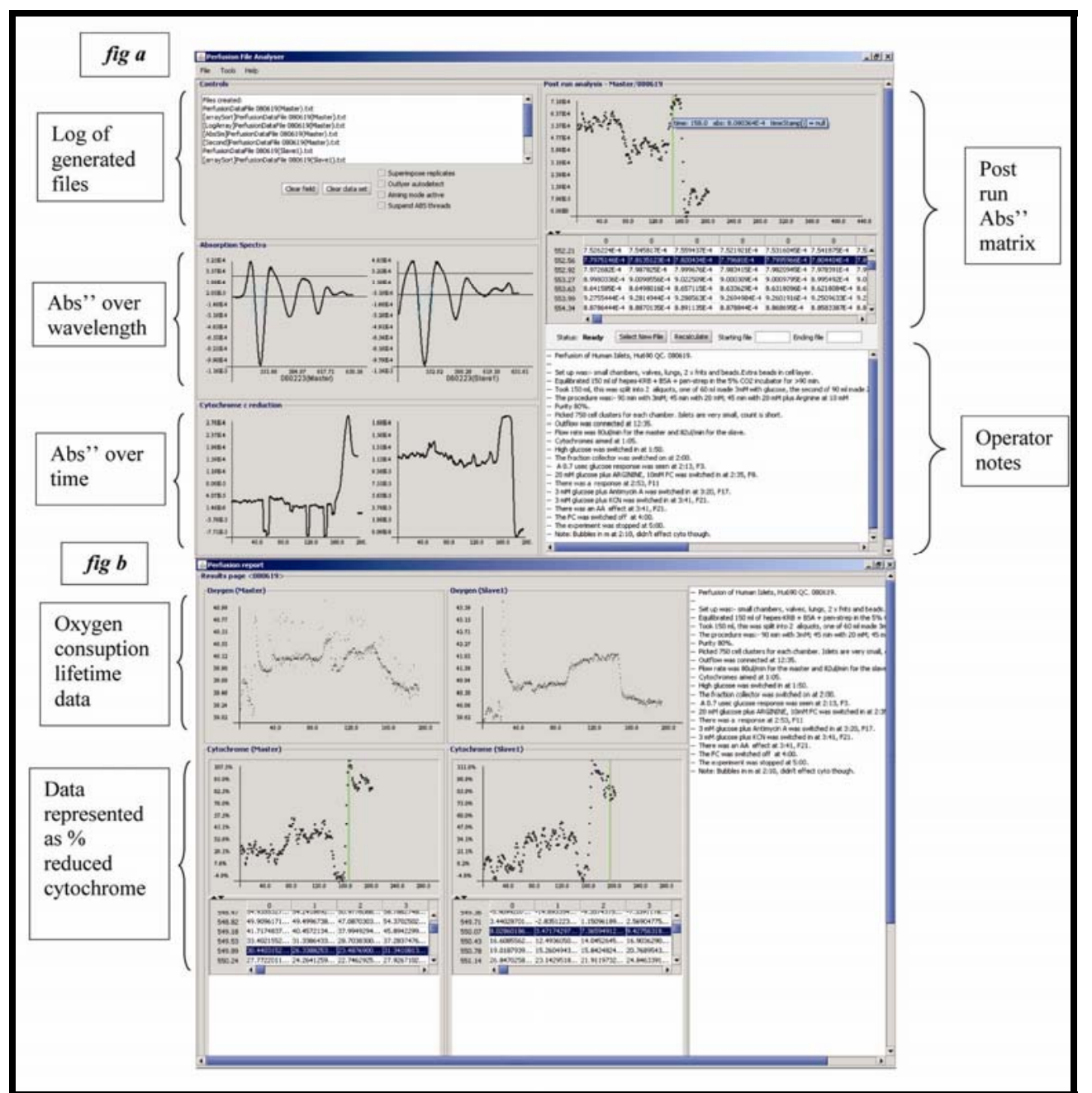

Figure 1: Perfusion file analyzer. (a) real time spectra over wavelength and time, and post run ABS" data; (b) Report page showing \% reduced cytochome c, oxygen consumption lifetimes, and operator notes.

\section{Caveat and future development:}

Currently, the program does not support saving of application-specific files, which would be useful for referencing previously calculated and annotated data sets. Also, PFA will only display data for one replicate prior to generating a report. Future versions of the PFA will save files for later 
reference and support tabbed display for multiple replicates in post analysis. In addition, because of the sensitivity of the assay, data sets often include outliers which, in some cases, can interfere with the calculation of Abs" ${ }_{\mathrm{AMA}}$ and Abs" ${ }_{\mathrm{KCN}}$ averages. An outlier detection algorithm is currently in development to address this issue as well.

References:

[01] I. R. Sweet et al., Diabetes, 53: 401 (2004) [PMID: 14747291]

[02] A. G. Cavinato et al., Anal Chem., 62: 1977 (1990) [PMID: 2240577]

[03] T. Kashiwagura et al., J Cell Pysiol., 120: 13 (1984) [PMID: 6330133]

Edited by P. Kangueane

Citation: Orr et al., Bioinformation 3(2): 65-67 (2008) License statement: This is an open-access article, which permits unrestricted use, distribution, and reproduction in any medium, for non-commercial purposes, provided the original author and source are credited.

\section{Supplemenatry material}

Equation 1

$$
A b s^{\prime \prime}=\frac{\Delta\left(\frac{\Delta A b s}{\Delta v}\right)}{\Delta v}
$$

Equation $1 \quad \mathrm{Cyto}_{\mathrm{c}}=100 *\left(\frac{\mathrm{Abs"}^{\prime \prime}}{\mathrm{Abs"}_{\mathrm{KCN}}}\right)$

$$
\mathrm{Abs}=\log \left(\frac{\text { intensity }- \text { intensity }_{\mathrm{bkg}}}{\text { intensity }_{\text {ref }}-\text { intensity }_{\mathrm{bkg}}}\right)
$$

